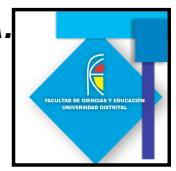

\title{
PROPUESTA DIDÁCTICA PARA RESOLUCIÓN DE PROBLEMAS DE FÍSICA POR INVESTIGACIÓN DIRIGIDA: UN ENFOQUE PARA FOMENTAR LA CULTURA CIENTÍFICA EN ESTUDIANTES DE GRADO DÉCIMO DEL INSTITUTO TÉCNICO RAFAEL REYES
}

\section{A DIDACTIC PROPOSAL FOR SOLVING PROBLEMS IN PHYSICS BY A CONDUCTED RESEARCH: AN APPROACH TO PROMOTE SCIENTIFIC CULTURE IN TENTH GRADE STUDENTS AT THE INSTITUTO TECNICO RAFAEL REYES}

Adriana Del Pilar Vega Pinzón ${ }^{1}$

\section{Resumen.}

El objetivo de este trabajo de investigación es determinar y valorar en que medida la planificación, implementación y evaluación de una propuesta didáctica de resolución de problemas de física por investigación dirigida, en la unidad temática de cinemática, posibilita el fomento de la cultura científica en estudiantes de grado decimo del Instituto Técnico Rafael Reyes.

La implementación de la propuesta se ha realizado durante dos años, con la participación de aproximadamente 120 estudiantes de grado decimo en la asignatura de Física durante el años 2009, 2010 y 2011.El diseño que se utilizo es pre-post, antes y después de la exposición a la intervención experimental, basado en la medición y comparación de actitudes hacia las Ciencias Físicas y el trabajo científico; aprendizajes con comprensión y la capacidad de resolver problemas, en los estudiantes.

Los resultados obtenidos muestran la validez de las hipótesis, demostrando que con la implementación del modelo de enseñanza por investigación dirigida se superan muchas de las deficiencias de la enseñanza tradicional en cuanto al fracaso y se fomenta la cultura científica en cuanto al mejoramiento de motivación, interés, aprendizajes significativos y capacidad para resolver problemas en la asignatura de física.

Palabras clave. Aprendizaje por investigación, cultura científica, cambio conceptual, cinemática, argumentación.

\footnotetext{
${ }^{1}$ Instituto Técnico Rafael Reyes -Duitama., Adriaveg73@yahoo.com
} 


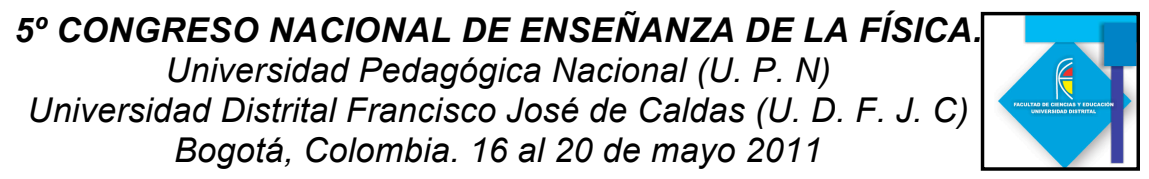

Abstract.

Bogotá, Colombia. 16 al 20 de mayo 2011

The objective of this research work is to indentify an assess how the planning, implementation and evaluation of a didactic proposal physics problem resolutions for research conducted, in the thematic unity of kinematics, enables the promotion of the scientific culture in students implementation and evaluation of a didactic proposal physics problem resolutions for research conducted, in the thematic unity of kinematics, enables the promotion of the scientific culture in students of tenth grade of Instituto Tecnico Rafael Reyes.

he implementation of the proposal has been made for two years, involving about 120 students in tenth grade in the course of Physics for the years 2009, 2010 and 2011. The design that was used is pre-post, before and after the explosion to the experimental intervention, based on the measurement and comparison of attitudes toward physical science and scientific work, learning with understanding and the ability to solve problems in students.

The obtained results show the validity of the hypothesis, showing that with the implementation of the teaching model for directed research are overcome many deficiencies of the traditional education in terms of failure and the scientific culture is fomented in terms of improvement of motivation, interest, significant learning and problem solving in the course of physics.

Keywords. Research learning, scientific culture, conceptual change, kinematics, scientific culture, argumentation.

\section{INTRODUCCION Y PLANTEAMIENTO DEL PROBLEMA}

Muchas de las investigaciones en didáctica de las ciencias y específicamente en Ciencias Físicas de nivel secundaria han arrojado resultados en torno a las dificultades de los estudiantes en el aprendizaje de ellas y como consecuencia su fracaso académico y la desmotivación; Una de las reflexiones que se ha generado gira en torno a cuestionar el modelo de enseñanza tradicional en el que el maestro transmite conceptos, teorías, leyes, ecuaciones de fenómenos físicos, como conceptos acabados y terminados. (Decir lo que es, o explicar como se hace, de una manera directa y acabada, junto con la realización de ejercicios (Beccerra,2006))

El papel del estudiante en este proceso es pasivo, limitándose a aceptar y memorizar conocimientos sin realizar análisis, ni interpretaciones de los fenómenos físicos, de manera que se ha constatado que el conocimiento de este objeto de estudio es ajeno al sujeto que aprende (Segura, 1997).

Al mismo tiempo muchas investigaciones han arrojado resultados que consideran que para que el aprendizaje de las ciencias sea significativo deben producirse cambios conceptuales entre las ideas intuitivas y las ideas científicas, y teniendo en cuenta que los procesos de aceptación y producción de los conocimientos cotidianos son diferentes a los procesos de aceptación y producción de los conocimientos científicos, entonces estos cambios 


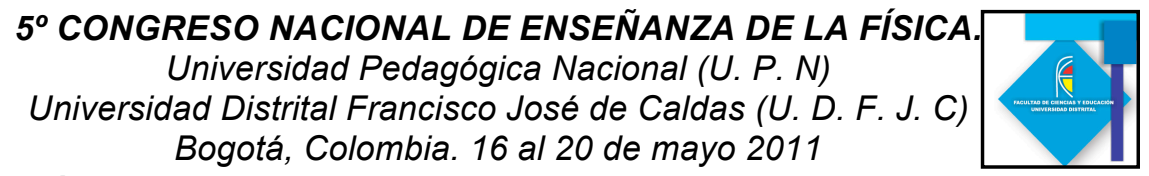

conceptuales están indisolublemente ligados a los cambios metodológicos, epistemológicos y actitudinales, tanto en docentes como en estudiantes (Lopera, 2002).

\begin{abstract}
En definitiva, este modelo de enseñanza de las ciencias se enmarca en el paradigma de enseñanza como investigación (Gil, 1983), tiene en cuenta las concepciones alternativas de los estudiantes, persigue un cambio conceptual (Posner et al., 1982), metodológico (Gil y Carrascosa, 1985a; Gil, 1986; Hashweh, 1986) y actitudinal (Aikenhead, 1985; Solbes y Vilches, 1989), para lo cual utiliza los aspectos esenciales del trabajo científico como forma de ejercitar la creatividad de manera rigurosa y contrastable(TORREGROSA, 2005).
\end{abstract}

Para producir estos cambios significativos en los procesos de enseñanza y aprendizaje de las ciencias Físicas específicamente en el: mejoramiento de actitudes, aprendizajes conceptuales y la capacidad de resolver problemas en los estudiantes se planifico, se implemento y se desarrollo la enseñanza de la cinemática de los grados decimos del Instituto Técnico Rafael Reyes, con el modelo de investigación dirigida o enseñanza problematizada.

\title{
APRENDIZAJE DE LAS CIENCIAS COMO INVESTIGACION DIRIGIDA:
}

Este modelo de enfoque Constructivista, organiza la estructura de los temas y del curso identificando los problemas que originaron las teorías, para propiciar ambientes de trabajo científico y de motivación, en donde se discuta la relevancia e interés de las situaciones problemáticas y así planificar las estrategias de solución del problema en un contexto Hipotético-deductivo, rico en episodios de argumentación y justificación (Becerra, 2006).

Este ambiente exige una cuidadosa planificación por parte del maestro, presentándose mediante programas de actividades debidamente articuladas (plan de investigación); algunos de los aspectos que deben incluirse en el plan se enunciaran a continuación:

- La discusión del posible interés y relevancia de las situaciones propuestas.

- El estudio cualitativo, significativo, de las situaciones problemáticas abordadas.

- La invención de conceptos y emisión de hipótesis fundamentadas.

- La elaboración y puesta en práctica de estrategias de resolución, incluyendo, en su caso, el diseño y realización de montajes experimentales

- El análisis y comunicación de los resultados, comparándolos con los obtenidos por otros grupos de estudiantes y por la comunidad científica.

- La consideración de las posibles perspectivas: contribuyendo a su profundización y resaltando en particular las relaciones CTSA, que enmarcan el desarrollo científico.

- La realización de síntesis, resúmenes y recapitulaciones con características similares a los informes científicos (Lamas, 1998). 


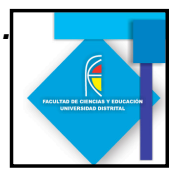

\section{PROCEDIMIENTO EXPERIMENTAL}

\section{Alumnos participantes}

La secuencia de enseñanza fue implementada en el contexto natural de una clase de Física; de modo que intervinieron todos los estudiantes de los cuatro grados decimos del Instituto Técnico Rafael Reyes. Los grupos estaban formados por 30 estudiantes cada uno, de clase social media-baja, con historiales académicos muy dispares en ciencias. Es preciso decir que los estudiantes en su gran mayoría estaban poco motivados por el estudio de las ciencias de manera que aproximadamente 6 de cada grupo pretendía estudiar ingeniería, los demás no querían continuar estudios que estuvieran relacionados con las ciencias.

\section{Implementación de la secuencia de enseñanza}

La experiencia se desarrolló durante doce semanas de tres horas semanales. Para ello, previamente se entregaba el plan de actividades para que en casa leyeran comprensivamente y desarrollaran las actividades, luego en clase los se organizaron en equipos de cuatro estudiantes donde expresaban sus aportes individuales y por medio

de trabajo cooperativo analizaban e interpretaban la información, intercambiaban ideas y opiniones; solicitaban ayuda al profesor, ante las dudas u obstáculos que les surgían, y, elaboraban una respuesta consensuada a las cuestiones planteadas. Luego, en mesa redonda socializaban sus respuestas, exponiendo al grupo sus conclusiones de manera que se generaba un debate en torno a los diferentes cuestionamientos, para llegar a las respuestas mas adecuadas.

El profesor moderaba estas discusiones introduciendo las correcciones y las orientaciones oportunas, según las necesidades de cada momento, y asumiendo un papel más activo cuando la situación lo requería. El propósito era llegar a las conclusiones argumentadas y con el máximo acuerdo posible. Permanentemente se estimulaba en los alumnos la autorregulación del aprendizaje por medio de la consignación de sus comentarios reflexivos sobre lo aprendido en clase (García-Carmona, 2005)

Es importante que en las memorias de los estudiantes consignadas en los cuadernos se indiquen las dificultades encontradas, los compromisos de mejoramiento, los errores cometidos, las correcciones elaboradas a partir de la interacciones entre compañeros y profesor, la evolución de su aprendizaje, etc 


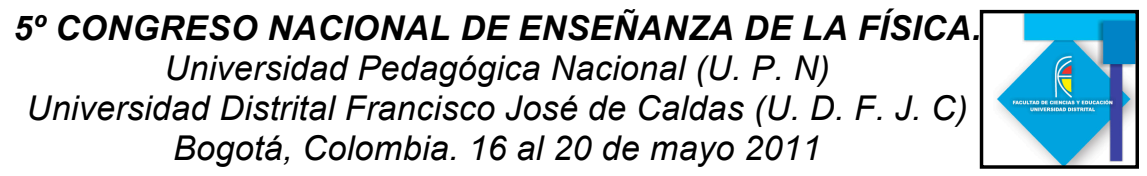

\section{Fragmento del plan de actividades de conceptos cinemáticas}

CONCEPTOS PARA DESCRIBIR LOS MOVIMENTOS

Es importante aclarar que estos conceptos son elaboraciones mentales del físico, que han sido construidas por la necesidad teórica o practica ante problemas de la descripción de movimientos.

PROBLEMA 3: ¿QUE CONCEPTOS FISICOS PODEMOS UTILIZAR PARA DESCRIBIR UN MOVIMIENTO CUALQUIERA?

Con el propósito de resolver nuestro problema, plantearemos una situación que genera curiosidad y asombro en muchos de nosotros.

1. ¿QUÉ OCURRE DUANTE EL MOVIMIENTO DE DESPEGUE DE UN AVIÓN? RELATIVIDAD DEL MOVIMIENTO

1.1. ¿Por qué vemos objetos en movimiento cuando están en reposo u objetos en reposo cuando están en movimiento?

A.1. Todos los pasajeros están esperando que el avión se empiece a mover. Imagina el avión cuando esta en estado de reposo en el aeropuerto:

Si estas en el aeropuerto en la pista de despegue ¿en que estado lo observas?

Si te encuentras en el Sol ¿en que estado lo observaras?

A.2. Y empieza el despegue: Imagina el pasajero Timoteo sentado en el interior del avión que despega:

¿En que estado vera la azafata a Timoteo?

¿En qué estado vera a Timoteo; una persona que se encuentra en la pista de despegue?

Si Timoteo deja caer libremente una esfera dentro del avión. ¿Cómo es la línea o curva descrita en el espacio por tal esfera, vista por la azafata?

Si estas en la pista de despegue y pudieras ver el interior del avión ¿Cómo verías la línea o curva descrita en el espacio por la esfera que dejo caer Timoteo? Ilustre la situación.

A.3. ¿Que concluyes de estas actividades?

\section{Fragmento del plan de actividades sobre emisión y comprobación de Hipótesis.}

A.57. Partiendo de las observaciones de la realidad, experiencias cotidianas e informaciones de diferentes medios: ¿podremos analizar la velocidad con la que cae un objeto solido en un medio viscoso y de un objeto que cae por un plano inclinado?.

Formula Hipótesis sobre el tipo de movimiento que pueden tener estos dos movimientos y en cada caso como es la variación del espacio recorrido, la velocidad y la aceleración en función del tiempo.

A.58.Proponga tres diseños experimentales en donde un objeto se mueva con velocidad constante, otro en el que aumente su velocidad y otro en el que disminuya su velocidad; de manera que permita comprobar relaciones de posición en función del tiempo.

A.59. Realiza los experimentos propuestos: Elabora una tabla de datos, grafica la posición del objeto en función del tiempo, encuentra la ecuación que relaciona la posición con el tiempo, realiza un análisis dimensional y concluye.

A.60. Analiza los resultados de los experimentos realizados y decide si se cumplen las hipótesis formuladas.

Presenta un informe detallado sobre el trabajo realizado, en el que se destaca cada una de las fases del trabajo científico: Planteamiento del problema, formulación de hipótesis, diseños experimentales, datos obtenidos, análisis de datos y conclusiones 


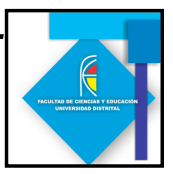

\section{CONCLUSIONES Y PERSPECTIVAS}

Con los resultados de este trabajo de investigación en donde se promueve el aprendizaje por investigación, podemos decir al finalizar el primer periodo muchas de las ideas previas que tenia el estudiante evolucionaron conceptualmente, la mayoría de los estudiantes

cambiaron su forma de percibir las ciencias Físicas para pasar de una asignatura matematizada a una asignatura en donde el trabajo científico es el eje que articula el aprendizaje de la Física. El plan de actividades estimulo la participación activa en los estudiantes, en donde las posibilidades de argumentar y contra-argumentar los cuestionamientos, los motivaron en todo el proceso de enseñanza y aprendizaje a que reflexionaran y auto -regularan su aprendizaje.

Los estudiantes manifestaron que con esta metodología las posibilidades de aprendizaje se ampliaban; porque el trabajo individual mas el trabajo grupal y luego el trabajo modelado por el maestro les permitía detectar individualmente sus errores conceptuales y así producir una cambios conceptuales.

Finalmente, esta investigación nos ha permitido hacer una reflexión sobre nuestro quehacer pedagógico, pues nos dio la posibilidad de replantear muchas actividades que cotidianamente se llevaban a cabo dentro del aula y que sus resultados no eran los mejores en términos académicos y de motivación. Esta experiencia nos muestra a los maestros de ciencias la necesidad de implicarnos en el fomento de la Cultura científica para todos.

\section{Referencias Bibliográficas}

Gil-Pérez, D. Macedo, B., Martínez-Torregrosa, J.; Sifredo, C.; Valdés, P. Y Vilches, A. (2005). ¿Cómo promover el interés por la cultura científica? Una propuesta fundamentada para la educación científica de jóvenes de 15 a 18 años, Década de la Educación para el Desarrollo Sostenible. UNESCO- OREALC

Becerra-Labra, C., Grás-Martí, A. Y Martínez Torregrosa, J. (2005). Revista Brasileira de Ensino de Física, 27(2), pp. 299-310. 